\title{
Defect structures in a layer-by-layer photonic band-gap crystal
}

\author{
E. Özbay \\ Department of Physics, Bilkent University, Ankara 06533, Turkey
}

\author{
G. Tuttle, M. Sigalas, C. M. Soukoulis, and K. M. Ho \\ Microelectronics Research Center and Ames Laboratory, Iowa State University, Ames, Iowa 50011
}

(Received 26 September 1994; revised manuscript received 16 January 1995)

\begin{abstract}
We have experimentally and theoretically investigated defect structures that are incorporated into a three-dimensional layer-by-layer photonic band-gap crystal. The defects are formed by either adding or removing dielectric material to or from the crystal. For both cases, we observed localized modes with frequencies that lie within the forbidden band gap of the pure crystal. Relatively high peak transmission (10 dB below the incident signal), and high quality factors (2000) have been measured. These measurements were in good agreement with theoretical simulations. Theoretical calculations also predict very high $\left(Q>10^{6}\right)$ quality factors for certain cavity structures.
\end{abstract}

It is now known that the propagation of electromagnetic (EM) waves in periodic dielectric arrays can be completely forbidden for a certain range of frequencies. ${ }^{1,2}$ These three-dimensional arrays-photonic band-gap (PBG) crystals - offer the potential to engineer the properties of the radiation field within these structures. ${ }^{3,4}$ The initial interest in this subject came from the proposal to use PBG crystals to inhibit spontaneous emission in photonic devices, leading to more efficient light emitters like thresholdless semiconductor lasers and single-mode light-emitting diodes. ${ }^{5-7}$ However, the difficulties of fabricating smaller scale structures restricted the experimental demonstration of the early PBG crystals to microwave frequencies $(12-15 \mathrm{GHz})$. Recently, we designed a three-dimensional layer-by-layer structure, which alleviates some of the fabrication difficulties inherent with the earlier designs. ${ }^{8}$ The layer-by-layer structure exhibits a sizable and robust photonic band gap over a wide range of structural parameters. Using a large-scale model made of cylindrical alumina rods, we confirmed the existence of a full photonic band gap at $K u$-band frequencies $(12-14 \mathrm{GHz}) .^{9}$ Using semiconductor macromachining techniques, we subsequently fabricated smaller scale structures with photonic band-gap frequencies ranging from 100 to $500 \mathrm{GHz} .^{10-12}$ This performance puts the new structure in the frequency range where a number of millimeter and submillimeter wave applications have been proposed, including efficient millimeter wave antennas, filters, sources, and waveguides. ${ }^{13-16}$ However, most of these applications are based on the presence of defect or cavity modes, which are obtained by locally disturbing the periodicity of the photonic crystal. ${ }^{17-19}$ The frequencies of these modes lie within the forbidden band gap of the pure crystal, and the associated fields are localized around the defect. In this paper, we demonstrate the existence of such cavity structures built around the layerby-layer PBG crystal.

The structure, shown schematically in Fig. 1, is constructed of layers of dielectric rods. The stacking sequence repeats every four layers, corresponding to a sin- gle unit cell in the stacking direction. In this investigation of defect structures, we followed the approach of Yablonovitch et al. ${ }^{17}$ wherein we either removed portions of the dielectric lattice or inserted extra dielectric materials into the air gaps. The basis for our study of defects was a large-scale model of our photonic structure, constructed of commercially available cylindrical alumina rods (refractive index $=3.1$ ). The rods were $0.318 \pm 0.013 \mathrm{~cm}$ in diameter and $15.24 \mathrm{~cm}$ long and were stacked with center-to-center separation of $1.123 \mathrm{~cm}$, chosen to obtain a dielectric filling ratio of $\sim 0.26$. This photonic crystal exhibits a full three-dimensional band gap between 11.7 and $13.5 \mathrm{GHz} .^{9}$

We first investigated the properties of defect structures formed by removing a portion of a single rod, as illustrated in Fig. 2(a). The length of the missing section is $d$, and to facilitate possible comparison to similar PBG crystals with different lattice constants, we define the defect

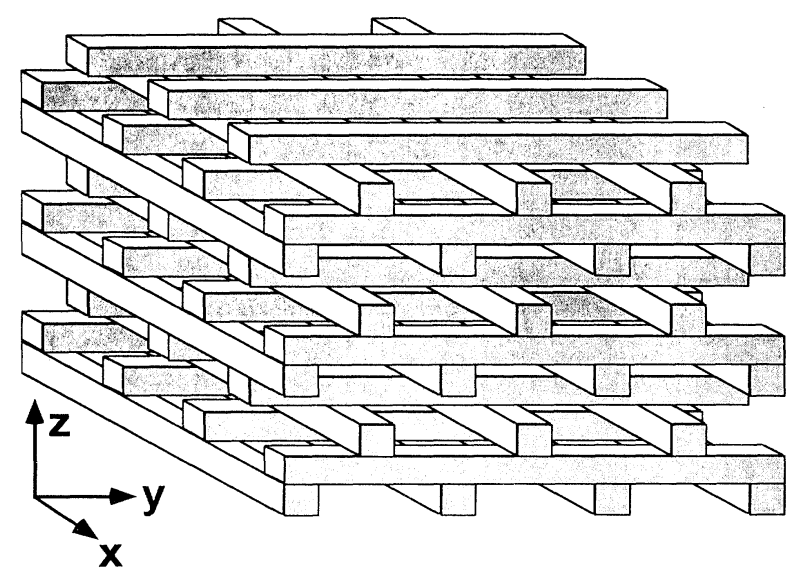

FIG. 1. This figure schematically illustrates the design of the three-dimensional photonic band-gap crystal. The structure is built by an orderly stacking of dielectric rods and repeats every four layers in the stacking direction. 
volume ratio $d / a$ where $a$ is the center-to-center separation between rods of the PBG crystal. We measured the transmission and phase dispersion properties of structures with defects using a Hewlett-Packard 8510A network analyzer with standard gain horn antennas to transmit and receive the EM radiation. The surroundings of the test set up were covered with absorber material to create an anechoic environment with a measurement a sensitivity of approximately $70 \mathrm{~dB}$.

Figure 2(b) shows the transmission characteristics of propagation along the $z$ axis (the stacking direction) for a structure with 4 unit cells (16 layers) along the $z$ axis. The defect was placed at the eighth layer of the structure and had a $d / a$ ratio of 1 . The electric field polarization vector of the incident EM wave e was parallel to the rods of the defect layer. The lower edge of the band gap along this propagation direction starts at $10.7 \mathrm{GHz}$, while the upper edge is around $17.4 \mathrm{GHz}$. The defect transmission was centered at $12.85 \mathrm{GHz}$, and the peak transmission was $30 \mathrm{~dB}$ below the incident signal. Using an expanded frequency scale, we measured the $Q$ factor (quality factor, defined as the center frequency divided by the peak's full width at half maximum) of the peak to be greater than 1000. We also measured the defect transmission at different incidence angles by rotating the sample with respect to the antennas while keeping e parallel to the rods of the defect layer. Figure 2 (c) shows the transmission properties of the same defect structure when the sample was rotated $35^{\circ}$. Although the upper and lower edges of the gap changed significantly due to different propagation directions, the defect frequency remained the same $(12.85 \mathrm{GHz})$. We also measured cavity structures with different $d / a$ ratios. Figure 2(d) shows the dependence of the defect frequency on the $d / a$ ratio. As can be seen from the plot, the peak frequency changed less than $2 \%$ as the defect volume changed by a factor of 10. We also found that the transmission via the defect was polarization dependent - no defect mode was present when e was perpendicular to the rods of the defect layer.

We used the transfer-matrix method (TMM), recently introduced by Pendry and MacKinnon, ${ }^{20}$ to calculate the EM transmission through a photonic crystal with defects. In the TMM method, the total volume of the photonic crystal is divided in small cells and the fields in each cell are coupled with those in the neighboring cells. Then the transfer matrix can be defined by relating the incident fields on one side of the PBG structure with the outgoing fields on the other side. Using TMM, the band structure of an infinite periodic system can be calculated, but the main advantage of this method is for the calculation of transmission and reflection coefficients for EM waves of various frequencies incident on a finite thickness slab of the PBG material. In that case, the material is assumed to be periodic in the directions parallel to the interfaces. The TMM method has previously been applied to defects in two-dimensional photonic band-gap structures, ${ }^{21}$ infinite PBG crystals in which the dielectric constants are complex and frequency dependent, ${ }^{22}$ and two-dimensional metallic PBG structures. ${ }^{23}$ In all these examples, the agreement between theoretical predictions and experiment measurements was very good.

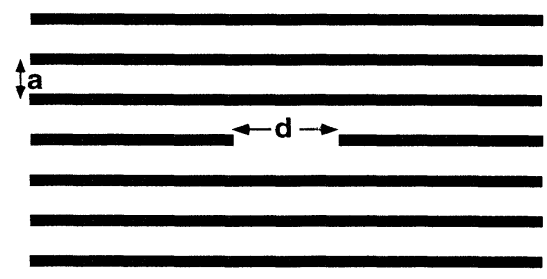

(a)

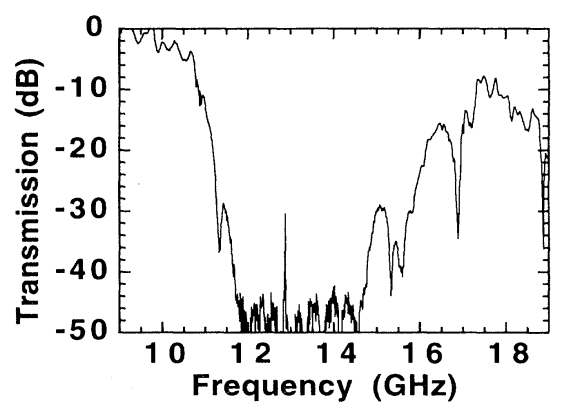

(b)

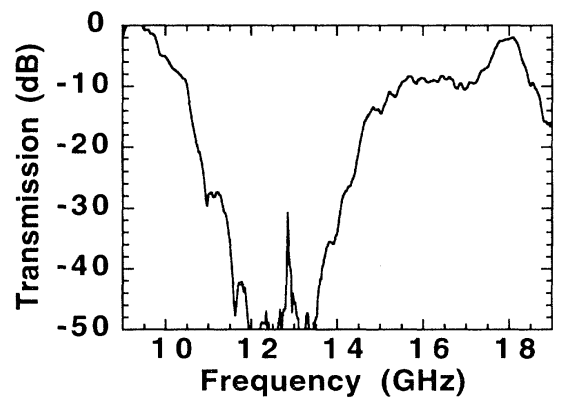

(c)

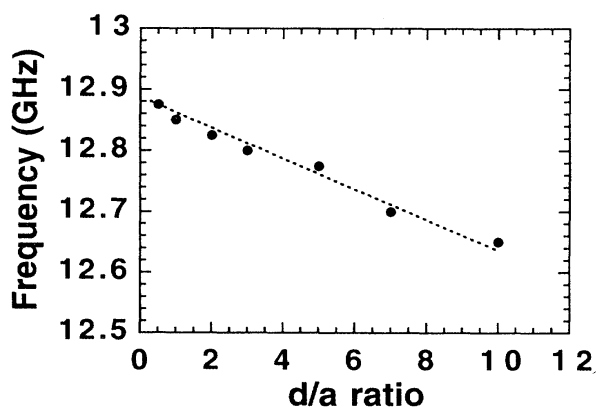

(d)

FIG. 2. (a) Missing material defect structure is obtained by removing a part of the alumina rod. The removed part has a width of $d$, while the center-to-center separation between two adjacent rods is $a$. (b) Transmission characteristics (along the stacking direction) obtained from a defect structure with a $d / a$ ratio of 1 . (c) Transmission characteristics of the same defect structure when it is rotated $35^{\circ}$. The defect frequency remains constant at different angles. (d) Defect mode frequency as a function of $d / a$ ratio. The total change in defect frequency is less than $2 \%$. 
Since the TMM method requires periodicity in the directions parallel to the interfaces, we examined the case of a periodic defect, made by removing every other from a single layer [see Fig. 3(a)]. The experiments are done using a structure consisting of 4 unit cells (16 layers), where the eighth layer is chosen as the defect layer. Figure 3(b) compares the predicted theoretical transmission with the experimental results. As can be seen from the plot, the agreement between theory and experiment is especially good for the defect frequency. The measured defect frequency is $12.62 \mathrm{GHz}$, while theory predicts $12.58 \mathrm{GHz}$. Again, we observed no defect modes when e is perpendicular to the defect rods. This is in accordance with theoretical results, which show that the peak transmission for the waves with e parallel to the removed rods is three orders of magnitude higher than the peak transmission obtained from the other polarization. Figure 3(c) shows the characteristics of the cavity mode on an

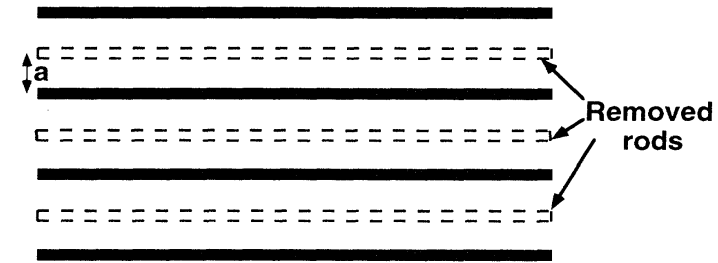

(a)

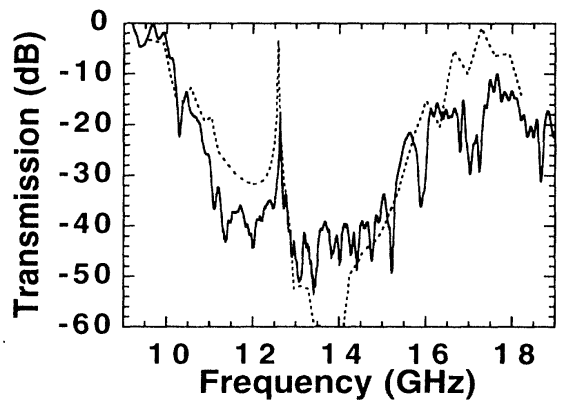

(b)

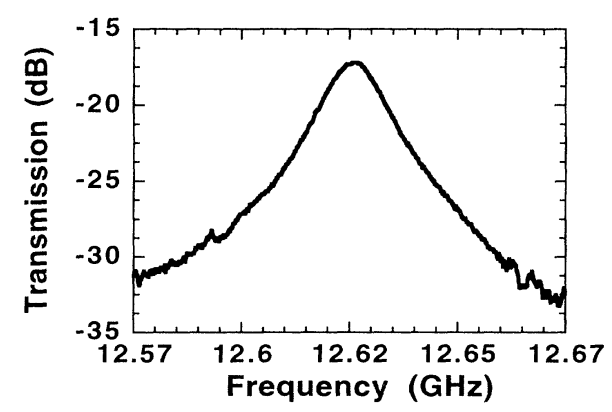

(c)

FIG. 3. (a) Every other rod of a single layer is removed to generate the defect layer. (b) Comparison of the theoretical (dashed line) and experimental (solid line) transmission characteristics of the defect structure. (c) Expanded frequency scale for the defect mode, which has a $Q$ factor of 2500 . expanded frequency scale. Although the measured $Q$ factor (750) of the cavity mode is very close to the theoretical $Q$ factor (800), the measured peak transmission of $-17 \mathrm{~dB}$ is far below the predicted value of $-4 \mathrm{~dB}$. The discrepancy is probably due to the finite length of the rods of the test structure, while our theoretical calculations assumed a crystal made of infinitely long rods. Calculations show that the defect frequency and $Q$ factor are not very sensitive to separation between the defects. In particular, the defect frequency increases from 12.58 to 12.68 to $12.39 \mathrm{GHz}$ as separation between the removed rods increases from $2 a$ to $3 a$ to $4 a$. Also, the $Q$ factor increases rapidly for increasing thickness of the PBG crystal. For a structure with 8 unit cells (32 layers), where the defect-layer structure shown in Fig. 3(a) is chosen as the 16th layer, calculations predict a defect frequency at $12.61 \mathrm{GHz}$ with a $Q$ factor more than $1.4 \times 10^{6}$. This suggests the possibility of building cavity structures with

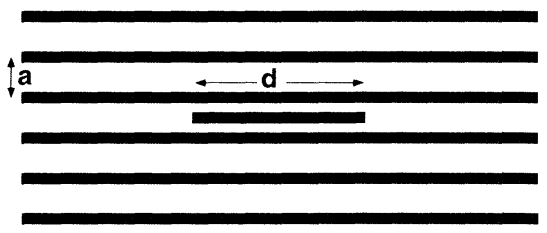

(a)

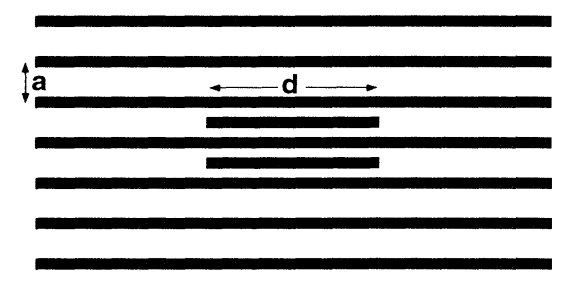

(b)

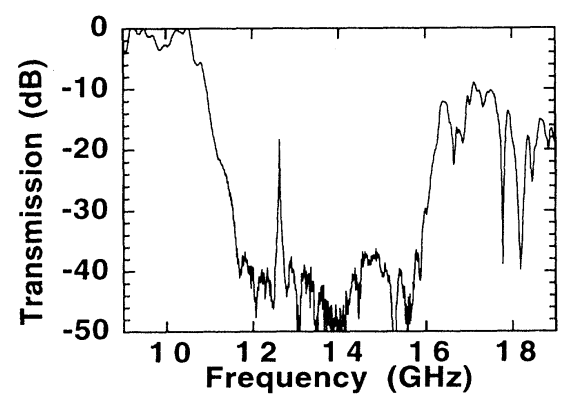

(c)

FIG. 4. (a) The defect structure obtained by a single rod. This defect structure did not yield any measurable defect mode. (b) The defect layer obtained by two rods of equal length. (c) Transmission characteristics of the defect structure depicted in (b). 
very high $Q$ factors from this geometry.

We also investigated defects that are obtained by adding pieces of rods or whole rods into the empty space of the PBG crystal. Figure 4(a) depicts the defect layer built by adding a single piece of dielectric material between two rods. Although we tried a number of different combinations (different defect lengths, different number of units cells), we did not observe any defect-mode transmission along the stacking direction. This could be due to limited coupling of the defect mode to the incident signal, which may result in a defect mode that is well below the attenuation within the photonic band-gap region. We were more successful when we added two pieces to create the defect, as shown in Fig. 4(b). Figure 4(c) shows the transmission characteristics for propagation in the $z$ direction (stacking direction) for a structure with 3 unit cells (12 layers) along the $z$ axis. The defects were added in the fifth layer of the structure and both rods had a $d / a$ ratio of 2. The defect transmission was at $12.6 \mathrm{GHz}$ with a peak transmission of $15 \mathrm{~dB}$ below the incident signal and $Q$ factor of $\sim 1000$. We again found that the defect frequency remained constant at different propagation directions. It should be noted that the transmission frequency measured for the additive defect is lower than the frequency of the subtractive defect. Yablonovitch et al. found an opposite trend for the defects in their crystal structure. ${ }^{17}$ This discrepancy is due to the different defect structures we have used in this paper. In our experiments, the defect structures are obtained by removing or adding cylindrical rods of the same radius but different length. When we extend our calculations for defects obtained by changing the radius of the cylinders, we find a similar trend obtained by Ref. 17. For these defects, transmission frequency measured for the additive defect is higher than the frequency of the subtractive defect. Yablonovitch et al. have named such defects as acceptors (missing material) or donors (added material) in analogy to the different doping types of the semiconductors. ${ }^{17} \mathrm{We}$ have found that this trend was not valid for certain defect structures, and we tried to avoid such a nomenclature, which may be confusing to readers.

Finally, we used TMM to study a periodic additive defect. Figure 5(a) depicts the periodic defect layer, in which an extra dielectric rod was inserted halfway between each lattice rod in one layer of the PBG crystal. This defect layer was the fifth layer of a structure that consisted of 3 unit cells (12 layers total). Theoretical and experimental spectra for transmission in the $z$ direction are compared in Fig. 5(b). The experimental $(12.4 \mathrm{GHz})$ and theoretical $(12.5 \mathrm{GHz})$ defect frequencies are in good agreement along with experimental and theoretical peak

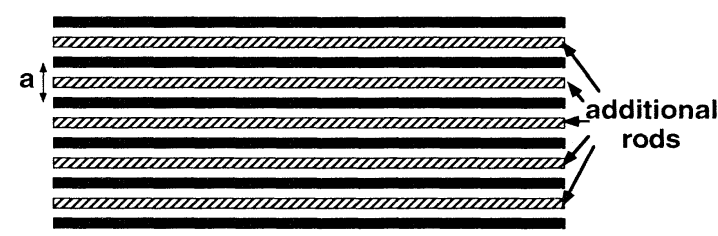

(a)

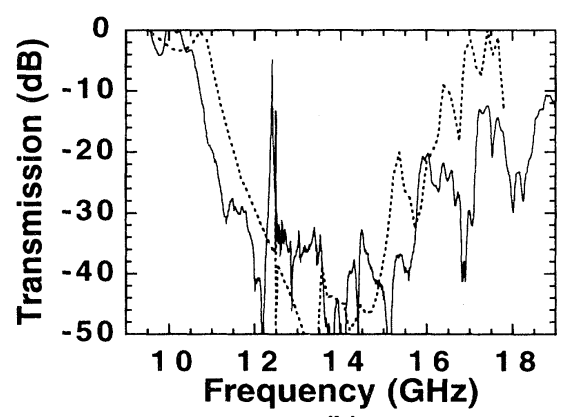

(b)

FIG. 5. (a) The defect layer is built by additional rods that are placed at the midpoints of adjacent rods. (b) Comparison of the theoretical (dashed line) and experimental (solid line) transmission characteristics of the defect structure.

transmissions, which are both $\sim 10 \mathrm{~dB}$ lower than the incident signal. However, theory predicts large $Q$ values ( $>10000$ ) while the measured $Q$ values are approximately 2000. Again, the difference is likely due to the infinite size of the experimental PBG structure.

In summary, we have investigated defect structures built around a rod-based photonic band-gap crystal. We have observed localized defect modes with high peak transmission and high $Q$ values. The measurements are in good agreement with our theoretical simulations. The availability of such defect structures will be a key tool for the proposed applications of PBG materials.

The authors would like to thank R. Biswas and C. T. Chan for helpful discussion and comments, and $\mathrm{D}$. Turner for his help in making the TMM code run on the nCube parallel processor. Ames Laboratory is operated for the U.S. Department of Energy by Iowa State University under Contract No. W-7405-Eng-82. This work was supported by the Director for Energy Research, Office of Basic Energy Sciences and Advanced Energy Projects, the Center for Advanced Technology Development at ISU, and the Scalable Computing Laboratory, which is funded by ISU and Ames Laboratory.
${ }^{1}$ E. Yablonovitch, T. J. Gmitter, and K. M. Leung, Phys. Rev. Lett. 67, 2295 (1991).

${ }^{2}$ K. M. Ho, C. T. Chan, and C. M. Soukoulis, Phys. Rev. Lett. 65, 3152 (1990).

${ }^{3}$ For a recent review, see the articles in Photonic Band Gaps and Localization, edited by C. M. Soukoulis (Plenum, New York,
1993).

${ }^{4}$ S. John and N. Akozbek, Phys. Rev. Lett. 71, 1168 (1993).

${ }^{5}$ D. L. Bullock, C. C. Shih, and R. S. Margulies, J. Opt. Soc. Am. B 10, 399 (1993).

${ }^{6}$ P. L. Gourley, J. R. Wendt, G. A. Vawter, T. M. Brennan, and B. E. Hammons, Appl. Phys. Lett. 6, 687 (1994). 
${ }^{7}$ J. P. Dowling, M. Scalora, M. J. Bleomer, and C. M. Bowden, J. Appl. Phys. 75, 1896 (1994).

${ }^{8}$ K. M. Ho, C. T. Chan, C. M. Soukoulis, R. Biswas, and M. Sigalas, Solid State Commun. 89, 413 (1994).

${ }^{9}$ E. Ozbay, A. Abeyta, G. Tuttle, M. Tringides, R. Biswas, C. Soukoulis, C. T. Chan, and K. M. Ho, Phys. Rev. B 50, 1945 (1994).

${ }^{10}$ E. Ozbay, E. Michel, G. Tuttle, M. Sigalas, R. Biswas, and K. M. Ho, Appl. Phys. Lett. 64, 2059 (1994).

${ }^{11}$ E. Ozbay, E. Michel, G. Tuttle, R. Biswas, K. M. Ho, J. Bostak, and D. M. Bloom, Opt. Lett. 19, 1155 (1994).

${ }^{12}$ E. Ozbay, G. Tuttle, M. Sigalas, R. Biswas, K. M. Ho, J. Bostak, and D. M. Bloom, Appl. Phys. Lett. 65, 1617 (1994).

${ }^{13}$ See the articles in J. Opt. Soc. Am. B 10 (1993), a special feature edited by C. M. Bowden, J. P. Dowling, and H. O. Everitt.

${ }^{14}$ E. R. Brown, C. D. Parker, and E. Yablonovitch, J. Opt. Soc. Am. B 10, 404 (1993).
${ }^{15}$ E. R. Brown, C. D. Parker, and O. B. McMahon, Appl. Phys. Lett. 64, 3345 (1994).

${ }^{16}$ C. J. Maggiore, A. M. Clogston, G. Spalek, W. C. Sailor, and F. M. Mueller, Appl. Phys. Lett. 64, 1451 (1994).

${ }^{17}$ E. Yablonovitch, T. J. Gmitter, R. D. Meade, A. M. Rappe, K. D. Brommer, and J. D. Joannopoulos, Phys. Rev. Lett. 67, 3380 (1991).

${ }^{18}$ S. L. McCall, P. M. Platzman, R. Dalichaouch, D. Smith, and S. Schultz, Phys. Rev. Lett. 67, 2017 (1991).

${ }^{19}$ K. M. Leung, J. Opt. Soc. Am. B 10, 303 (1993).

20J. B. Pendry and A. MacKinnon, Phys. Rev. Lett. 69, 2772 (1992); J. B. Pendry, J. Mod. Opt. 41, 209 (1994).

${ }^{21}$ M. M. Sigalas, C. M. Soukoulis, E. N. Economou, C. T. Chan, and K. M. Ho, Phys. Rev. B 48, 14121 (1993).

${ }^{22}$ M. M. Sigalas, C. M. Soukoulis, C. T. Chan, and K. M. Ho, Phys. Rev. B 49, 11080 (1994).

${ }^{23}$ D. R. Smith, S. Shultz, N. Kroll, M. Sigalas, K. M. Ho, and C. M. Soukoulis, Appl. Phys. Lett. 65, 645 (1994). 


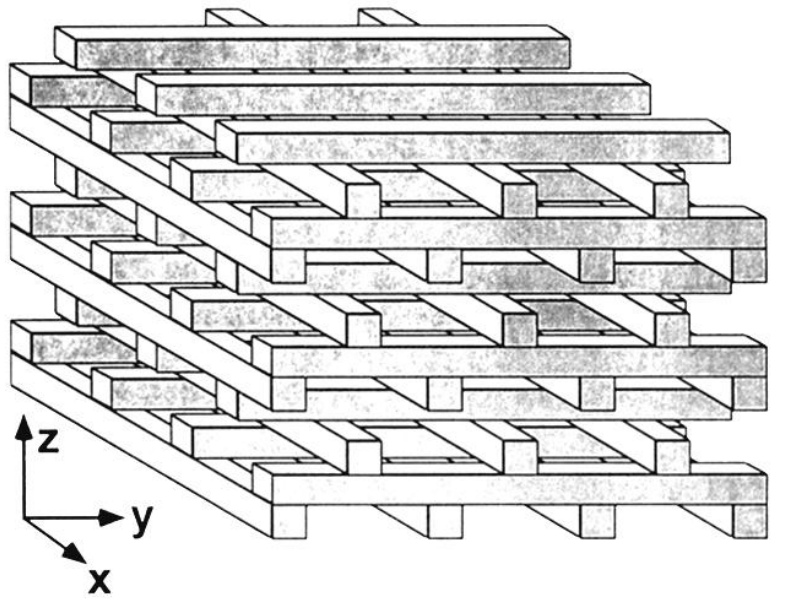

FIG. 1. This figure schematically illustrates the design of the three-dimensional photonic band-gap crystal. The structure is built by an orderly stacking of dielectric rods and repeats every four layers in the stacking direction. 